Article

\title{
General Blaschke Bodies and the Asymmetric Negative Solutions of Shephard Problem
}

\author{
Tian $\mathrm{Li}^{1}$, Weidong Wang ${ }^{2, *}$ and Yaping Mao ${ }^{1, *}$ \\ 1 College of Mathematics and Statistics, Qinghai Normal University, Xining 810000, China \\ 2 Department of Mathematics, China Three Gorges University, Yichang 443002, China \\ * Correspondence: wangwd722@163.com (W.W.); maoyaping@ymail.com (Y.M.)
}

Received: 11 June 2019; Accepted: 4 July 2019; Published: 10 July 2019

Abstract: In this article, based on the Blaschke combination of convex bodies, we define the general Blaschke bodies and obtain the extremal values of their volume and affine surface area. Further, we study the asymmetric negative solutions of the Shephard problem for the projection bodies.

Keywords: general Blaschke body; extremal value; Shephard problem; asymmetric negative solution

PACS: 52A20; 52A39; 52A40

\section{Introduction and Main Results}

Mixed volume and surface area measure are the core of the classical Brunn-Minkowski theory. Based on the surface area measure, the Blaschke combination of convex bodies is defined (see [1]) as follows: for $M, N \in \mathcal{K}^{n}, n \geq 2$ and $\alpha, \beta \geq 0$ (not both zero), the Blaschke combination $\alpha \odot M \mp \beta \odot N$ of $M$ and $N$ satisfies

$$
S(\alpha \odot M \mp \beta \odot N, \cdot)=\alpha S(M, \cdot)+\beta S(N, \cdot) .
$$

Here $\mathcal{K}^{n}$ denotes the set of convex bodies which are convex and compact subsets with non-empty interiors in the Euclidean space $\mathbb{R}^{n}, S(M, \cdot)$ denotes the surface area measure of $M \in \mathcal{K}^{n}$ (see [1,2]), the operation ' $\mp$ ' is called Blaschke addition, $\alpha \odot M$ denotes Blaschke scalar multiplication and $\alpha \odot M=\alpha^{\frac{1}{n-1}} M$.

Put $\alpha=\beta=\frac{1}{2}$ and $N=-M$ in (1), the Blaschke body, $\nabla M$, of $M \in \mathcal{K}^{n}$ is given by

$$
\nabla M=\frac{1}{2} \odot M \mp \frac{1}{2} \odot(-M) .
$$

Based on the Blaschke bodies, we define a family of Blaschke bodies with a parameter which is called general Blaschke bodies. For $M \in \mathcal{K}^{n}, n \geq 2$ and $\gamma \in[-1,1]$, the general Blaschke body, $\nabla^{\gamma} M$, of $M$ is defined by

$$
S\left(\nabla^{\gamma} M, \cdot\right)=g_{1}(\gamma) S(M, \cdot)+g_{2}(\gamma) S(-M, \cdot),
$$

where

$$
g_{1}(\gamma)=\frac{(1+\gamma)^{2}}{2\left(1+\gamma^{2}\right)}, \quad g_{2}(\gamma)=\frac{(1-\gamma)^{2}}{2\left(1+\gamma^{2}\right)}
$$

By (4), we get that

$$
\begin{gathered}
g_{1}(\gamma)+g_{2}(\gamma)=1, \\
g_{1}(-\gamma)=g_{2}(\gamma), \quad g_{2}(-\gamma)=g_{1}(\gamma) .
\end{gathered}
$$


Combining with (3), it easily yields

$$
\nabla^{\gamma} M=g_{1}(\gamma) \odot M \mp g_{2}(\gamma) \odot(-M) .
$$

Moreover, by (2), (4) and (7), $\nabla^{0} M=\nabla M$ when $\gamma=0 ; \nabla^{+1} M=M$ and $\nabla^{-1} M=-M$ when $\gamma= \pm 1$.

In this article, we study general Blaschke bodies and their applications. First, the extremum of the volume and affine surface area for the general Blaschke bodies were established by us. Further, as the applications of the extreme values, we give the asymmetric negative forms for the Shephard problem of projection bodies.

For the extremal values of the volume of general Blaschke bodies, we give the following result.

Theorem 1. Suppose $M \in \mathcal{K}^{n}$ and $\gamma \in[-1,1]$, then

$$
V(\nabla M) \geq V\left(\nabla^{\gamma} M\right) \geq V(M) .
$$

If $\gamma \neq 0$, then equality holds in the left inequality if and only if $M$ is centrally symmetric; if $\gamma \neq \pm 1$, then equality holds in the right inequality if and only if $M$ is also centrally symmetric. Here $V(M)$ is the n-dimensional volume of a body $M$.

Meanwhile, according to the affine surface area of convex bodies (see (18)), we obtain another type of extremum for general Blaschke bodies.

Theorem 2. Suppose $M \in \mathcal{K}^{n}$ and $\gamma \in[-1,1]$, then

$$
\Omega(\nabla M) \geq \Omega\left(\nabla^{\gamma} M\right) \geq \Omega(M) .
$$

If $\gamma \neq 0$, then equality holds in the left inequality if and only if $M$ is centrally symmetric; if $\gamma \neq \pm 1$, then equality holds in the right inequality if and only if $M$ is also centrally symmetric. Here $\Omega(M)$ is the affine surface area of $M \in \mathcal{K}^{n}$.

The notion of projection bodies was introduced at the turn of the previous century by Minkowski. For a convex body $Q$, the projection body $\Pi Q$ of $Q$ is the origin-symmetric convex body whose support function is defined by (see [1])

$$
h_{\Pi Q}\left(\varepsilon_{1}\right)=V_{n-1}\left(Q \mid \varepsilon_{1}^{\perp}\right)=\frac{1}{2} \int_{S^{n-1}}\left|\varepsilon_{1} \cdot \varepsilon_{2}\right| d S\left(Q, \varepsilon_{2}\right)
$$

for all $\varepsilon_{1}, \varepsilon_{2} \in S^{n-1}\left(S^{n-1}\right.$ is the unit sphere in $\left.\mathbb{R}^{n}\right)$. Here $V_{n-1}$ denotes the $(n-1)$-dimensional volume.

The projection body is an extremely important notion in Brunn-Minkowski theory. For many results on the projection bodies, see [3-12] or books [1,2]. In particular, Petty [9] and Schneider [11] gave the following negative solutions for the Shephard problems of projection bodies, respectively.

Theorem $3([9,11])$. Let $N \in \mathcal{K}^{n}$. If $N$ is not centrally symmetric, then there exists $M \in \mathcal{K}_{o s}^{n}$ satisfying $\Pi M \subset \Pi N$, but

$$
V(M)>V(N) .
$$

Here $\mathcal{K}_{\text {os }}^{n}$ is the set of origin-symmetric convex bodies in $\mathbb{R}^{n}$.

Theorem $4([9,11]) . \quad L e t ~ N \in \mathcal{K}^{n}$. If $N$ is not centrally symmetric, then there exists $M \in \mathcal{K}_{o s}^{n}$ satisfying $\Pi M \subset \Pi N$, but

$$
\Omega(M)>\Omega(N) .
$$


Evidently, the negative solutions are origin-symmetric convex bodies in Theorems 3 and 4 . Here, as the applications of Theorems 1 and 2, we give the asymmetric negative solutions for the Shephard problems of projection bodies, namely, we extend the scope of negative solutions in Theorems 3 and 4 from $M \in \mathcal{K}_{o s}^{n}$ to $M \in \mathcal{K}^{n}$, respectively.

Theorem 5. Let $N \in \mathcal{K}^{n}$. If $N$ is not centrally symmetric, then there exists $M \in \mathcal{K}^{n}$ satisfying $\Pi M \subset$ $\Pi N$, but

$$
V(M)>V(N)
$$

Theorem 6. Let $N \in \mathcal{K}^{n}$. If $N$ is not centrally symmetric, then there exists $M \in \mathcal{K}^{n}$ satisfying $\Pi M \subset$ $\Pi N$, but

$$
\Omega(M)>\Omega(N)
$$

Our works are part of a new and rapidly evolving asymmetric Brunn-Minkowski theory. For the researches of asymmetric Brunn-Minkowski theory, see [13-43].

In this article, the proofs of Theorems 1 and 2 shall be completed in Section 4 and the proofs of Theorems 5 and 6 shall be given in Section 5. In Section 3, we show some properties of general Blaschke bodies.

\section{Preliminaries}

\subsection{Mixed Volume and Surface Area Measure}

The support function of a convex body $Q$ can be defined by (see [1,2])

$$
h(Q, m)=\max \{m \cdot n: n \in Q\}, m \in \mathbb{R}^{n},
$$

here $m \cdot n$ is the standard inner product for $m$ and $n$.

The mixed volume of $Q_{1}, Q_{2}, \cdots, Q_{n} \in \mathcal{K}^{n}$ is defined by (see [1])

$$
V\left(Q_{1}, Q_{2}, \cdots, Q_{n}\right)=\frac{1}{n} \int_{S^{n-1}} h\left(Q_{n}, \varepsilon\right) d S\left(Q_{1}, Q_{2}, \cdots, Q_{n-1}, \varepsilon\right), \varepsilon \in S^{n-1},
$$

here $S\left(Q_{1}, Q_{2}, \cdots, Q_{n-1}, \cdot\right)$ is called the mixed surface area measure of $Q_{1}, Q_{2}, \cdots, Q_{n-1}$.

Let $B$ denote the standard unit ball in $\mathbb{R}^{n}$ and its volume is written by $\omega_{n}=V(B)$. The mixed surface area measure $S(Q, \cdots, Q, B, \cdots, B, \cdot)$, with $n-j-1$ copies of $Q$ and $j$ copies of $B$, is usually written by Lutwak (see [44])

$$
S_{j}(Q, \cdot)=S(Q, n-j-1 ; B, j, \cdot), j=0,1, \cdots, n-1
$$

In particular, let $j=0$, then the surface area measure, $S(Q, \cdot)$, of $Q \in \mathcal{K}^{n}$ is given by

$$
S(Q, \cdot)=S_{0}(Q, \cdot)=S(Q, n-1 ; B, 0, \cdot)
$$

Put $Q_{1}=Q_{2}=\cdots=Q_{n-j}=M, Q_{n-j+1}=\cdots=Q_{n}=N$ in (12). Then the volume $V\left(Q_{1}, Q_{2}, \cdots, Q_{n}\right)$ is written by

$$
V\left(Q_{1}, Q_{2}, \cdots, Q_{n}\right)=V(M, n-j ; N, j)=V_{j}(M, N)
$$

here $V(M, n-j ; N, j)$ contains $n-j$ copies of $M$ and $j$ copies of $N$.

From (12), (13), and (14), if $j=1$, then for arbitrary $\varepsilon \in S^{n-1}$,

$$
V_{1}(M, N)=\frac{1}{n} \int_{S^{n-1}} h(N, \varepsilon) d S(M, \varepsilon)
$$


In [45], Lutwak showed that the mixed volume and surface area measure have the translation property, i.e., for any $m, n \in \mathbb{R}^{n}$,

$$
\begin{gathered}
V_{1}(M+m, N+n)=V_{1}(M, N), \\
S(M+m, \cdot)=S(M, \cdot) .
\end{gathered}
$$

Using the mixed volumes, the following result can be obtained

Theorem 7 ([2]). Let $M, N \in \mathcal{K}^{n}$. If all $Q \in \mathcal{K}^{n}$ and

$$
V_{1}(M, Q)=V_{1}(N, Q),
$$

then $M$ and $N$ are translates of each other.

By (15) and Theorem 7, we easily get that

Theorem 8. Let $M, N \in \mathcal{K}^{n}$. If any $\varepsilon \in S^{n-1}$ and

$$
S(M, \varepsilon)=S(N, \varepsilon)
$$

then $M$ and $N$ are translates of each other.

\subsection{Affine Surface Area}

For $Q \subseteq \mathbb{R}^{n}$ is non-empty, the polar set, $Q^{*}$, of $Q$ is a convex set defined as (see [1])

$$
Q^{*}=\left\{m \in \mathbb{R}^{n}: m \cdot n \leq 1, \text { for all } n \in Q\right\} .
$$

The notion of classical affine surface area was proposed by Leichtwei $\beta$ (see [46]). For the set of star bodies with the origin in $\mathbb{R}^{n}$, we write $\mathcal{S}_{0}^{n}$. Define the affine surface area, $\Omega(M)$, of $M \in \mathcal{K}^{n}$ by

$$
n^{-\frac{1}{n}} \Omega(M)^{\frac{n+1}{n}}=\inf \left\{n V_{1}\left(M, N^{*}\right) V(N)^{\frac{1}{n}}: N \in \mathcal{S}_{o}^{n}\right\} .
$$

\section{Some Properties of General Blaschke Bodies}

In this section, we will establish the following properties of general Blaschke bodies.

Theorem 9. Suppose $M \in \mathcal{K}^{n}$ and $\gamma \in[-1,1]$, then

(i) $\nabla^{-\gamma} M=\nabla^{\gamma}(-M)$;

(ii) $\nabla^{\gamma}(-M)$ is a translation of $-\nabla^{\gamma} M$, i.e.,

$$
\nabla^{\gamma}(-M)=-\nabla^{\gamma} M+m, m \in \mathbb{R}^{n} .
$$

Proof. (i) Combining with (6) and (7), we deduce that for $\gamma \in[-1,1]$,

$$
\nabla^{-\gamma} M=g_{1}(-\gamma) \odot M \mp g_{2}(-\gamma) \odot(-M)=g_{2}(\gamma) \odot M \mp g_{1}(\gamma) \odot(-M)=\nabla^{\gamma}(-M) .
$$

(ii) From (3), for any $\varepsilon \in S^{n-1}$, we have that

$$
\begin{aligned}
S\left(-\nabla^{\gamma} M, \varepsilon\right) & =S\left(\nabla^{\gamma} M,-\varepsilon\right)=g_{1}(\gamma) S(M,-\varepsilon)+g_{2}(\gamma) S(-M,-\varepsilon) \\
& =g_{1}(\gamma) S(-M, \varepsilon)+g_{2}(\gamma) S(M, \varepsilon)=S\left(\nabla^{\gamma}(-M), \varepsilon\right) .
\end{aligned}
$$


Using (15), for any $Q \in \mathcal{K}^{n}$, we obtain

$$
\begin{aligned}
V_{1}\left(-\nabla^{\gamma} M, Q\right) & =\frac{1}{n} \int_{S^{n-1}} h(Q, \varepsilon) d S\left(-\nabla^{\gamma} M, \varepsilon\right) \\
& =\frac{1}{n} \int_{S^{n-1}} h(Q, \varepsilon) d S\left(\nabla^{\gamma}(-M), \varepsilon\right) \\
& =V_{1}\left(\nabla^{\gamma}(-M), Q\right) .
\end{aligned}
$$

Therefore, from Theorem $7, \nabla^{\gamma}(-M)$ is a translation of $-\nabla^{\gamma} M$. In other words,

$$
\nabla^{\gamma}(-M)=-\nabla^{\gamma} M+m, m \in \mathbb{R}^{n} .
$$

This yields Theorem 9 .

Theorem 10. Let $M \in \mathcal{K}^{n}$ and $\gamma \in[-1,1]$. If $\gamma \neq 0$, then $\nabla^{\gamma} M$ is centrally symmetric if and only if $M$ is centrally symmetric.

Proof. According to (3) and the proof of (20), we see that for any $\varepsilon \in S^{n-1}$,

$$
\begin{gathered}
S\left(\nabla^{\gamma} M, \varepsilon\right)=g_{1}(\gamma) S(M, \varepsilon)+g_{2}(\gamma) S(-M, \varepsilon), \\
S\left(-\nabla^{\gamma} M, \varepsilon\right)=g_{1}(\gamma) S(-M, \varepsilon)+g_{2}(\gamma) S(M, \varepsilon) .
\end{gathered}
$$

If $M$ is centrally symmetric, i.e., $M=-M+m, m \in \mathbb{R}^{n}$, then from (5), (17), (21), and (22), we have

$$
S\left(\nabla^{\gamma} M, \varepsilon\right)=S\left(-\nabla^{\gamma} M, \varepsilon\right) .
$$

So by Theorem 8 , it yields $\nabla^{\gamma} M=-\nabla^{\gamma} M+m$. Namely, $\nabla^{\gamma} M$ is centrally symmetric.

On the contrary, if $\nabla^{\gamma} M$ is centrally symmetric, i.e., $\nabla^{\gamma} M=-\nabla^{\gamma} M+m$, then from (21) and (22), we obtain

$$
\left[g_{1}(\gamma)-g_{2}(\gamma)\right] S(M, \varepsilon)=\left[g_{1}(\gamma)-g_{2}(\gamma)\right] S(-M, \varepsilon) .
$$

Since $g_{1}(\gamma)-g_{2}(\gamma) \neq 0$ when $\gamma \neq 0$, thus it means that $S(M, \varepsilon)=S(-M, \varepsilon)$ for any $\varepsilon \in S^{n-1}$. This and Theorem 8 yield that $M=-M+m, m \in \mathbb{R}^{n}$. Namely, $M$ is centrally symmetric.

\section{The Extremal Values of General Blaschke Bodies}

In this section, Theorems 1 and 2 will be proved by us. The proof of Theorem 1 requires the following Lemma.

Lemma 1 ([47]). If $M, N \in \mathcal{K}^{n}, n \geq 2$ and $\alpha, \beta \geq 0$ (not both zero), then

$$
V(\alpha \odot M \mp \beta \odot N)^{\frac{n-1}{n}} \geq \alpha V(M)^{\frac{n-1}{n}}+\beta V(N)^{\frac{n-1}{n}},
$$

with equality if and only if $M$ and $N$ are homothetic.

Proof of Theorem 1. Using (7), (23) and (5), we deduce that for any $\gamma \in[-1,1]$,

$$
\begin{aligned}
V\left(\nabla^{\gamma} M\right)^{\frac{n-1}{n}} & =V\left(g_{1}(\gamma) \odot M \mp g_{2}(\gamma) \odot(-M)\right)^{\frac{n-1}{n}} \\
& \geq g_{1}(\gamma) V(M)^{\frac{n-1}{n}}+g_{2}(\gamma) V(-M)^{\frac{n-1}{n}} \\
& =V(M)^{\frac{n-1}{n}} .
\end{aligned}
$$


Hence, we have for $n \geq 2$,

$$
V\left(\nabla^{\gamma} M\right) \geq V(M) .
$$

This yields the right inequality of (8).

Evidently, (24) is an identity if $\gamma= \pm 1$. Moreover, if $\gamma \neq \pm 1$, then from the equality condition of (23), the equality holds in (24) if and only if $M$ and $-M$ are homothetic, it means $M$ is a centrally symmetric convex body. Namely, the sufficient and necessary condition of equality holds in the right inequality of (8) is that $M$ shall be centrally symmetric if $\gamma \neq \pm 1$.

Now, we complete the proof of left inequality in (8). Combining with (1), (3), (5) and (6), we obtain that

$$
\begin{aligned}
& S\left(\nabla^{\gamma} M, \varepsilon\right)+S\left(\nabla^{-\gamma} M, \varepsilon\right) \\
= & {\left[g_{1}(\gamma) S(M, \varepsilon)+g_{2}(\gamma) S(-M, \varepsilon)\right]+\left[g_{1}(-\gamma) S(M, \varepsilon)+g_{2}(-\gamma) S(-M, \varepsilon)\right] } \\
= & {\left[g_{1}(\gamma)+g_{1}(-\gamma)\right] S(M, \varepsilon)+\left[g_{2}(\gamma)+g_{2}(-\gamma)\right] S(-M, \varepsilon) } \\
= & S(M, \varepsilon)+S(-M, \varepsilon)=2 S(\nabla M, \varepsilon)
\end{aligned}
$$

for arbitrary $\varepsilon \in S^{n-1}$. Thus,

$$
S(\nabla M, \varepsilon)=\frac{1}{2} S\left(\nabla^{\gamma} M, \varepsilon\right)+\frac{1}{2} S\left(\nabla^{-\gamma} M, \varepsilon\right) .
$$

By (1), (2) and Theorem 9, it yields

$$
\begin{aligned}
\nabla M & =\frac{1}{2} \odot \nabla^{\gamma} M \mp \frac{1}{2} \odot \nabla^{-\gamma} M \\
& =\frac{1}{2} \odot \nabla^{\gamma} M \mp \frac{1}{2} \odot \nabla^{\gamma}(-M) \\
& =\frac{1}{2} \odot \nabla^{\gamma} M \mp \frac{1}{2} \odot\left(-\nabla^{\gamma} M+m\right), \quad m \in \mathbb{R}^{n} .
\end{aligned}
$$

Using (25) and (23), we obtain

$$
\begin{aligned}
V(\nabla M)^{\frac{n-1}{n}} & =V\left(\frac{1}{2} \odot \nabla^{\gamma} M \mp \frac{1}{2} \odot\left(-\nabla^{\gamma} M+m\right)\right)^{\frac{n-1}{n}} \\
& \geq \frac{1}{2} V\left(\nabla^{\gamma} M\right)^{\frac{n-1}{n}}+\frac{1}{2} V\left(-\nabla^{\gamma} M+m\right)^{\frac{n-1}{n}} \\
& =V\left(\nabla^{\gamma} M\right)^{\frac{n-1}{n}} .
\end{aligned}
$$

So for $n \geq 2$,

$$
V(\nabla M) \geq V\left(\nabla^{\gamma} M\right) .
$$

This yields the left inequality of (8).

Evidently, (26) is an identity if $\gamma=0$. If $\gamma \neq 0$, in accordance with the equality condition of (23), the equality holds in (26) if and only if $\nabla^{\gamma} M$ and $-\nabla^{\gamma} M+m$ are homothetic, this means $\nabla^{\gamma} M$ and $-\nabla^{\gamma} M$ are homothetic, i.e., $\nabla^{\gamma} M$ is a centrally symmetric convex body. Hence, together with Theorem 10, $M$ is a centrally symmetric convex body. It implies that equality holds in the left inequality of (8) if and only if $M$ is centrally symmetric if $\gamma \neq 0$.

Lemma 2 ([45]). Suppose $M, N \in \mathcal{K}^{n}$ and $\alpha, \beta \geq 0$ (not both zero), then

$$
\Omega(\alpha \odot M \mp \beta \odot N)^{\frac{n+1}{n}} \geq \alpha \Omega(M)^{\frac{n+1}{n}}+\beta \Omega(N)^{\frac{n+1}{n}},
$$

with equality if and only if $M$ and $N$ can be homothetic. 
Proof of Theorem 2. According to (7), (27) and (5), we obtain for arbitrary $\gamma \in[-1,1]$,

$$
\begin{aligned}
\Omega\left(\nabla^{\gamma} M\right)^{\frac{n+1}{n}} & =\Omega\left(g_{1}(\gamma) \odot M \mp g_{2}(\gamma) \odot(-M)\right)^{\frac{n+1}{n}} \\
& \geq g_{1}(\gamma) \Omega(M)^{\frac{n+1}{n}}+g_{2}(\gamma) \Omega(-M)^{\frac{n+1}{n}} \\
& =\Omega(M)^{\frac{n+1}{n}} .
\end{aligned}
$$

Hence

$$
\Omega\left(\nabla^{\gamma} M\right) \geq \Omega(M)
$$

The right inequality of (9) is proved.

From the equality condition of (27), the equality holds in (28) if and only if $M$ and $-M$ are homothetic if $\gamma \neq \pm 1$. That is to say, $M$ is a centrally symmetric convex body.

Further, we prove the left inequality of (9). By (16), we have for arbitrary $N \in \mathcal{S}_{0}^{n}$ and $m \in \mathbb{R}^{n}$,

$$
V_{1}\left(-\nabla^{\gamma} M+m, N^{*}\right)=V_{1}\left(-\nabla^{\gamma} M, N^{*}\right) .
$$

Then from (18), we get

$$
\Omega\left(-\nabla^{\gamma} M+m\right)=\Omega\left(-\nabla^{\gamma} M\right)
$$

Hence using (25) and (27), we obtain

$$
\begin{aligned}
\Omega(\nabla M)^{\frac{n+1}{n}} & =\Omega\left(\frac{1}{2} \odot \nabla^{\gamma} M \mp \frac{1}{2} \odot\left(-\nabla^{\gamma} M+m\right)\right)^{\frac{n+1}{n}} \\
& \geq \frac{1}{2} \Omega\left(\nabla^{\gamma} M\right)^{\frac{n+1}{n}}+\frac{1}{2} \Omega\left(-\nabla^{\gamma} M+m\right)^{\frac{n+1}{n}} \\
& =\Omega\left(\nabla^{\gamma} M\right)^{\frac{n+1}{n}} .
\end{aligned}
$$

Therefore

$$
\Omega(\nabla M) \geq \Omega\left(\nabla^{\gamma} M\right)
$$

This is just the left inequality of (9).

From the condition of equality holds in (27), the equality holds in (29) if and only if $\nabla^{\gamma} M$ and $-\nabla^{\gamma} M+m$ are homothetic if $\gamma \neq 0$. It means $\nabla^{\gamma} M$ and $-\nabla^{\gamma} M$ are homothetic, i.e., $\nabla^{\gamma} M$ is a centrally symmetric convex body. Therefore, together with Theorem $10, M$ is a centrally symmetric convex body. In other words, the equality holds in the left inequality of (9) if and only if $M$ is centrally symmetric if $\gamma \neq 0$.

\section{The Asymmetric Negative Solutions for Shephard Problem}

In this section, we give the volume and affine surface area forms of asymmetric negative solutions for the Shephard problems of projection bodies.

Lemma 3. If $Q \in \mathcal{K}^{n}$ and $\gamma \in[-1,1]$, then

$$
\Pi\left(\nabla^{\gamma} Q\right)=\Pi Q .
$$

Proof. Using (10) and (3), we infer that

$$
\begin{aligned}
h_{\Pi\left(\nabla^{\gamma} Q\right)}\left(\varepsilon_{1}\right) & =\frac{1}{2} \int_{S^{n-1}}\left|\varepsilon_{1} \cdot \varepsilon_{2}\right| d S\left(\nabla^{\gamma} Q, \varepsilon_{2}\right) \\
& =\frac{1}{2} \int_{S^{n-1}}\left|\varepsilon_{1} \cdot \varepsilon_{2}\right| d\left[g_{1}(\gamma) S\left(Q, \varepsilon_{2}\right)+g_{2}(\gamma) S\left(-Q, \varepsilon_{2}\right)\right]
\end{aligned}
$$




$$
=g_{1}(\gamma) h_{\Pi Q}\left(\varepsilon_{1}\right)+g_{2}(\gamma) h_{\Pi(-Q)}\left(\varepsilon_{1}\right) .
$$

Associated with (10), it follows that $\Pi(-Q)=\Pi Q$. Hence together with (31) and (5), we have for arbitrary $\varepsilon \in S^{n-1}$,

$$
h_{\Pi\left(\nabla^{\gamma} Q\right)}(\varepsilon)=h_{\Pi Q}(\varepsilon) .
$$

Namely, $\Pi\left(\nabla^{\gamma} Q\right)=\Pi Q$.

Proof of Theorem 5. Notice that $N$ is not centrally symmetric, so by Theorem 1 , when $\gamma \neq \pm 1$,

$$
V\left(\nabla^{\gamma} N\right)>V(N)
$$

Take small enough $0<\delta<1$ such that $V\left((1-\delta) \nabla^{\gamma} N\right)>V(N)$. Therefore, let $M=(1-\delta) \nabla^{\gamma} N$ (for $\gamma=0, M \in \mathcal{K}_{o s}^{n} ;$ for $\gamma \neq 0, M \in \mathcal{K}^{n} \backslash \mathcal{K}_{o s}^{n}$ ), then

$$
V(M)>V(N) .
$$

However using (30) and $\Pi(1-\delta) N=(1-\delta)^{n-1} \Pi N$, we conclude that

$$
\Pi M=\Pi\left((1-\delta) \nabla^{\gamma} N\right)=(1-\delta)^{n-1} \Pi\left(\nabla^{\gamma} N\right)=(1-\delta)^{n-1} \Pi N \subset \Pi N .
$$

Proof of Theorem 6. Because $N$ is not centrally symmetric, together with Theorem 2 , when $\gamma \neq \pm 1$,

$$
\Omega\left(\nabla^{\gamma} N\right)>\Omega(N)
$$

Put small sufficiently $0<\delta<1$ such that $\Omega\left((1-\delta) \nabla^{\gamma} N\right)>\Omega(N)$. Hence, let $M=(1-\delta) \nabla^{\gamma} N$ (for $\gamma=0, M \in \mathcal{K}_{o s}^{n}$; for $\gamma \neq 0, M \in \mathcal{K}^{n} \backslash \mathcal{K}_{o s}^{n}$ ), then

$$
\Omega(M)>\Omega(N) .
$$

Whereas, in the same spirit of the proof of Theorem 5, we have $\Pi M \subset \Pi N$.

Author Contributions: Conceptualization, T.L., W.W. and Y.M.; methodology, T.L., W.W. and Y.M.; software, T.L.; validation, T.L., W.W. and Y.M.; formal analysis, T.L. and W.W.; investigation, T.L. and W.W.; resources, W.W. and Y.M.; writing-original draft preparation, T.L.; writing-review and editing, T.L. and W.W.; supervision, W.W. and Y.M.; project administration, T.L., W.W. and Y.M.; funding acquisition, T.L., W.W. and Y.M..

Funding: Research is supported by the National Natural Science Foundation of China (No. 11371224, 11601254, 11551001, 11161037, 61763041, 11661068 and 11461054), the Science Found of Qinghai Province (No. 2016-ZJ-948Q and 2014-ZJ-907), the Qinghai Key Laboratory of Internet of Things Project (2017-ZJ-Y21) and the research foundation of Qinghai Normal University (2019zr002).

Acknowledgments: The authors would like to sincerely thank the referees for very valuable and helpful comments and suggestions, which made the paper more accurate and readable.

Conflicts of Interest: The authors declare no conflict of interest.

\section{References}

1. Gardner, R.J. Geometric Tomography, 2nd ed.; Cambridge University Press: Cambridge, UK, 2006.

2. Schneider, R. Convex Bodies: The Brunn-Minkowski Theory, 2nd ed.; Cambridge University Press: Cambridge, UK, 2014.

3. Bolker, E.D. A class of convex bodies. Trans. Am. Math. Soc. 1969, 145, 323-345. [CrossRef]

4. Bourgain, J.; Lindenstrauss, J. Projection bodies. In Geometric Aspects of Functional Analysis; Lecture Notes in Mathematics; Springer: Berlin, Germany, 1988; Volume 1317; pp. 250-270.

5. Brannen, N.S. Volumes of projection bodies. Mathematika 1996, 43, 255-264. [CrossRef]

6. Lutwak, E. Mixed projection inequalities. Trans. Am. Math. Soc. 1985, 287, 91-106. [CrossRef] 
7. Lutwak, E. On a conjectured projection inequality of Petty. Contemp. Math. 1990, 113, 171-181.

8. Lutwak, E. Inequalities for mixed projection bodies. Am. Math. Soc. 1993, 339, 901-916. [CrossRef]

9. Petty, C.M. Projection bodies, Proc Coll Convexty (Copenhagen 1965). Kobenhavns Univ. Math. Inst. 1967, 2, 234-241.

10. Petty, C.M. Isoperimetric problems. In Proceedings of the Conference on Convexity and Combinatorial Geometry; University of Oklahoma: Norman, OK, USA, 1972; pp. 26-41.

11. Schneider, R. Zu einem problem von Shephard über die projectionen konvexer körper. Mathematische Zeitschrift 1967, 101, 71-82. [CrossRef]

12. Zhang, G.Y. Restricted chord projection and affine inequalities. Geom. Dedicata 1991, 39, 213-222. [CrossRef]

13. Feng, Y.B.; Wang, W.D. General $L_{p}$-harmonic Blaschke bodies. Proc. Indian Acad. Sci. Math. Sci. 2014, 124, 109-119. [CrossRef]

14. Feng, Y.B.; Wang, W.D.; Lu, F.H. Some inequalities on general $L_{p}$-centroid bodies. Math. Inequal. Appl. 2015, 18, 39-49.

15. Haberl, C. $L_{p}$-intersection bodies. Adv. Math. 2008, 217, 2599-2624. [CrossRef]

16. Haberl, C.; Ludwig, M. A characterization of $L_{p}$ intersection bodies. Int. Math. Res. Not. 2006, $2006,10548$. [CrossRef]

17. Haberl, C.; Schuster, F.E. General $L_{p}$ affine isoperimetric inequalities. J. Differ. Geom. 2009, 83, 1-26. [CrossRef]

18. Haberl, C.; Schuster, F.E. Asymmetric affine $L_{p}$ Sobolev inequalities. J. Funct. Anal. 2009, 257, $641-658$. [CrossRef]

19. Haberl, C.; Schuster, F.E.; Xiao, J. An asymmetric affine Pólya-Szegö principle. Math. Ann. 2012, 352, 517-542. [CrossRef]

20. Li, T.; Wang, W.D. Some inequalities for asymmetric $L_{p}$-mean zonoids. Quaest. Math. 2019, 42, 631-649. [CrossRef]

21. Li, Z.F.; Wang, W.D. General $L_{p}$-mixed chord integrals of star Bodies. J. Inequal. Appl. 2016, $2016,58$. [CrossRef]

22. Li, Z.F.; Wang, W.D. Inequalities on asymmetric $L_{p}$-harmonic radial bodies. J. Nonlinear Sci. Appl. 2017, 10, 3612-3618. [CrossRef]

23. Ludwig, M. Projection bodies and valuations. Adv. Math. 2002, 172, 158-168. [CrossRef]

24. Ludwig, M. Minkowski valuations. Trans. Am. Math. Soc. 2005, 357, 4191-4213. [CrossRef]

25. Pei, Y.N.; Wang, W.D. Shephard type problems for general $L_{p}$-centroid bodies. J. Inequal. Appl. 2015, 2015, 287. [CrossRef]

26. Pei, Y.N.; Wang, W.D. A type of Busemann-Petty problems for general $L_{p}$-intersection bodies. Wuhan Univ. J. Nat. Sci. 2015, 20, 471-475. [CrossRef]

27. Schuster, F.E.; Weberndorfer, M. Volume inequalities for asymmetric Wulff shapes. J. Differ. Geom. 2012, 92, 263-283. [CrossRef]

28. Shen, Z.H.; Li, Y.N.; Wang, W.D. $L_{p}$-dual geominimal surface areas for the general $L_{p}$-intersection bodies. J. Nonlinear Sci. Appl. 2017, 10, 3519-3529. [CrossRef]

29. Shi, W.; Wang, W.D. Extremum values of asymmetric $L_{p}$-difference bodies for quermassintegrals and dual quermassintegrals. Wuhan Univ. J. Nat. Sci. 2018, 23, 283-288. [CrossRef]

30. Wan, X.Y.; Wang, W.D. Petty projection inequalities for the general $L_{p}$-mixed projection bodies. Wuhan Univ. J. Nat. Sci. 2012, 17, 190-194. [CrossRef]

31. Wang, J.Y.; Wang, W.D. General $L_{p}$-dual Blaschke bodies and the applications. J. Inequal. Appl. 2015, 2015, 233. [CrossRef]

32. Wang, W.D.; Feng, Y.B. A general $L_{p}$-version of Petty's affine projection inequality. Taiwan J. Math. 2013, 17, 517-528. [CrossRef]

33. Wang, W.D.; Li, T. Volume extremals of general $L_{p}$-centroid bodies. J. Math. Inequal. 2017, 11, $193-207$. [CrossRef]

34. Wang, W.D.; Li, Y.N. Busemann-Petty problems for general $L_{p}$-intersection bodies. Acta Math. Sin. 2015, 31, 777-786. [CrossRef]

35. Wang, W.D.; Li, Y.N. General $L_{p}$-intersection bodies. Taiwan. J. Math. 2015, 19, 1247-1259. [CrossRef]

36. Wang, W.D.; Ma, T.Y. Asymmetric $L_{p}$-difference bodies. Proc. Am. Math. Soc. 2014, 142, $2517-2527$. [CrossRef] 
37. Wang, W.D.; Wan, X.Y. Shephard type problems for general $L_{p}$-projection bodies. Taiwan J. Math. 2012, $16,1749-1762$.

38. Wang, W.D.; Wang, J.Y. Extremum of geometric functionals involving general $L_{p}$-projection bodies. J. Inequal. Appl. 2016, 2016, 135. [CrossRef]

39. Weberndorfer, M. Shadow systems of asymmetric $L_{p}$ zonotopes. Adv. Math. 2013, 240, 613-635. [CrossRef]

40. Wei, B.; Wang, W.D. Some inequalities for general $L_{p}$-harmonic Blaschke bodies. J. Math. Inequal. 2016, 10, 63-73. [CrossRef]

41. Yan, L.; Wang, W.D. The general $L_{p}$-mixed brightness integrals. J. Inequal. Appl. 2015, 2015, 190. [CrossRef]

42. Zhang, P.; Zhang, X.H.; Wang, W.D. The general $L_{p}$-dual mixed brightness integrals. Int. J. Appl. Math. 2017, 47, 138-142.

43. Zhou, Y.P. General $L_{p}$-mixed width-integral of convex bodies and related inequalities. J. Nonlinear Sci. Appl. 2017, 10, 4372-4380. [CrossRef]

44. Lutwak, E. The Brunn-Minkowski-Firey theory I: Mixed volumes and the Minkowski problem. J. Differ. Geom. 1993, 38, 131-150. [CrossRef]

45. Lutwak, E. Extended affine surface area. Adv. Math. 1991, 85, 39-68. [CrossRef]

46. Leichtwei $\beta$, K. Bemerkungen zur Definition einer erweiterten Affinoberfläche von E.Lutwak. Manuscr. Math. 1989, 65, 181-197.

47. Lutwak, E. Volume of mixed bodies. Trans. Amer. Math. Soc. 1986, 294, 487-500. [CrossRef]

(C) 2019 by the authors. Licensee MDPI, Basel, Switzerland. This article is an open access article distributed under the terms and conditions of the Creative Commons Attribution (CC BY) license (http://creativecommons.org/licenses/by/4.0/). 